\title{
溶胶一凝胶法制备超疏水性薄膜摩擦学性能的研究
}

\author{
万勇，张泉，李 杨 \\ (青岛理工大学 机械工程学院, 青岛 266033)
}

\begin{abstract}
摘 要: 采用溶胶一凝胶技术在铝表面涂覆氧化铝薄膜, 再利用长链脂肪酸对氧化铝薄膜进行疏水改性, 在金属铝 表面构筑了具有较强减摩性能的超疏水薄膜。研究了沸水及水合肼溶液处理对氧化铝薄膜表面微纳织构的影响; 探 讨了脂肪酸分子结构对薄膜静态和动态润湿性的影响, 利用球盘式微纳米摩擦磨损试验机评价了薄膜的摩擦学性 能。结果显示, 水合肼溶液处理后的氧化铝薄膜经硬脂酸改性后不仅表现出超疏水性能, 而且具有较强的减摩性 能。
\end{abstract}

关 键 词: 溶胶-凝胶; 铝; 超疏水性能; 减摩特性

中图分类号: O647 文献标识码: A

\section{Tribological Performance of Sol-Gel Derived Superhydrophobic Film}

\author{
WAN Yong, ZHANG Quan, LI Yang
}

(School of Mechanical Engineering, Qingdao Technological University, Qingdao 266033, China)

\begin{abstract}
A simple two-step process was developed in this study to render aluminum with lower friction and superhydrophobicity. Alumina film was firstly fabricated on aluminum by Sol-Gel technology. Fatty acid was then deposited on alumina film to endow superhydrophobicity. The surface morphology of alumina film after treatment using boiling water or hydrazine solution was evaluated. The effect of molecular structure of fatty acid on the wettability of the composite film was investigated. Friction-reducing behavior of the superhydrophobic films on aluminum was determined in a ball-on-plate configuration. It was found that the stearic acid film on alumina film treated by hydrazine solution showed superhydrophobicity and low friction, leading to significantly extending lifespan of the alumina film.
\end{abstract}

Key words: Sol-Gel; aluminum; superhydrophobicity; friction-reducing performance

金属铝由于比重小、质量轻，同时又具有良好 的金属延展性和可塑性，在日常生活和工业生产中 得到了广泛的应用。然而，铝合金由于化学活性高 和黏性强导致其耐磨和抗擦伤性能差，难于润滑， 特别是在常用的钢一铝摩擦副中, 由于铝和钢之间 存在较大的固溶度, 摩擦过程中易发生铝向钢的转 移，从而造成铝件的严重磨损; 在铝合金的加工过 程中, 这种转移会严重影响工件的表面质量, 缩短 模具的使用寿命 ${ }^{[1-4]}$ 。近年来, 随着铝基材料在数字
微镜器件等微机电系统(MEMS)中的应用, 材料表 面微纳尺寸上粘着失效和摩擦磨损严重影响 MEMS 的性能和稳定性 ${ }^{[5-7]}$ 。以往的研究发现, 传统意义上 的液体润滑剂已经不再适合润滑这类微纳机构, 而 环境中水分子在接触区的凝结所形成的毛细管力是 微纳接触条件下导致材料摩擦和磨损的主要因素。 因此, 利用表面改性技术实现表面疏水化以减小水 分子在表面的吸附是解决这一问题的重要手段。目 前多在材料表面沉积自组装单分子膜(SAMs)来实 
现表面疏水化, 从而减小材料的摩擦和磨损 ${ }^{[8-10]}$ 。但 在滑动接触条件下, SAMs 膜普遍存在稳定性差、耐 磨性能差和寿命有限等缺点, 无法满足对 MEMS 连 续润滑的要求。

近年来，受自然界中荷叶等多种植物的叶片、 蝴蝶等鳞翅目昆虫的翅膀、水鸟的羽毛等生物表 面结构的启发, 超疏水材料以其独特的润湿性能 引起科学界和工业界广泛关注 ${ }^{[11-17]}$ 。 $\operatorname{Ruan}^{[18]}$ 和 Farzaneh $^{[19-20]}$ 等发现，在铝合金表面构筑的超疏 水薄膜具有良好的防冰敷性能, Duan 等 ${ }^{[21]}$ 在铝合 金表面制备的层状复合金属氢氧化物超疏水薄膜 具有显著的防腐蚀性能。目前的研究均关注超疏 水薄膜的润湿性能, 尚未见薄膜在摩擦学领域, 特别是在 MEMS 润滑中应用研究的报道。另一方 面，尽管目前在超疏水薄膜的制备方法方面有大 量研究探索, 但仍没有达到简单可靠、成本低廉的 程度。

由于溶胶一凝胶技术生成的氧化物薄膜具有高 的热稳定性和机械耐久性 ${ }^{[22-25]}$, 本研究采用此技术 在铝表面构筑超疏水薄膜, 重点考察铝表面超疏水 薄膜的摩擦学特性, 以有效改善微纳尺度下铝基材 料的润滑, 并探索能够实现制备简单、适合大规模 生产的可靠方法。

\section{1 实验方法}

\section{1 氧化铝薄膜的制备}

把 1060 铝板裁剪成 $15 \mathrm{~mm} \times 15 \mathrm{~mm}$ 的方形片, 分别采用 400 目、 800 目和 1500 目砂纸打磨抛光, 采 用 $4 \mathrm{~mol} / \mathrm{L}$ 盐酸溶液处理 $30 \mathrm{~s}$, 去离子水和无水乙醇 中超声处理 $10 \mathrm{~min}$, 氮气吹干备用。

按文献 [26]报道方法在铝表面构筑氧化铝薄 膜。以六水合三氯化铝 $\left(\mathrm{AlCl}_{3} \cdot 6 \mathrm{H}_{2} \mathrm{O}\right.$, 分析纯)作为前 体化合物, 无水乙醇 $(\mathrm{EtOH}$, 分析纯)为溶剂, 乙酰丙 醞 $\left(\mathrm{CH}_{3} \mathrm{COCH}_{2} \mathrm{COCH}_{3}\right.$, 分析纯) 为稳定剂, 配制铝 溶胶。首先, 将三氯化铝和无水乙醇配制成相应浓 度的三氯化铝乙醇溶液, 在室温下高速摚拌, 同时 缓慢加入乙酰丙酮, 使体积比 $\left(\mathrm{AlCl}_{3} \cdot 6 \mathrm{H}_{2} \mathrm{O}\right.$ : EtOH : $\mathrm{CH}_{3} \mathrm{COCH}_{2} \mathrm{COCH}_{3}$ 为 $1: 20: 2$ ), 搅拌 $1 \mathrm{~h}$ 后 $50^{\circ} \mathrm{C}$ 水 浴再搅拌 $1 \mathrm{~h}$ 。配制好的铝溶胶呈橙黄色, 不易沉淀。

采用旋涂法在金属铝表面制备氧化铝薄膜。将 新制备好的铝溶胶 1 滴(约 $0.03 \mathrm{~mL}$ )滴加到样品表面, 在速度为 $550 \mathrm{r} / \mathrm{min}$ 下保持 $9 \mathrm{~s}$, 然后在 $1500 \mathrm{r} / \mathrm{min}$ 保持 $15 \mathrm{~s}$ 。旋涂结束后立即在 $60^{\circ} \mathrm{C}$ 下干燥 $10 \mathrm{~min}$ 。 镀膜过程重复三次。最后将样品置于马弗炉中, $480^{\circ} \mathrm{C}$
烧结 $90 \mathrm{~min}$, 随炉冷却后取出备用。

\section{2 氧化铝薄膜的疏水改性}

将获得的氧化铝薄膜分成三组。其中, 一组作 为参照组, 不做处理; 将另一组样品置于 $95^{\circ} \mathrm{C}$ 沸 水中处理 $10 \mathrm{~min}$; 将第三组样品浸入 $8 \%$ 的水合肼 溶液中, 室温(约 $25^{\circ} \mathrm{C}$ )下保持 $24 \mathrm{~h}$ 。分别用去离子 水以及无水乙醇冲洗, 氮气吹干, 待用。

室温下, 将经不同处理的氧化铝薄膜分别放入 $0.01 \mathrm{~mol} / \mathrm{L}$ 的脂肪酸(硬脂酸、油酸或月桂酸)的乙醇 溶液中, 静置 $24 \mathrm{~h}$ 。取出后分别用去离子水以及无 水乙醇冲洗，氮气吹干备用。

\section{3 薄膜的表征}

在室温 (约 $25^{\circ} \mathrm{C}$ ) 和相对湿度 $40 \%$ 50\%条件下， 用接触角测定仪 (CAM101, KSV Instruments LTD) 测量样品的水接触角。在每个样品上的不同位置测 量 5 个点, 取平均值。用扫描电子显微镜(SEM, 日 本 Hitachi S-3500N 型)观察刻蚀后的铝表面形貌。 采用傅里叶变换红外显微镜(IR, Nicolette iN10)对 铝基底上硬脂酸薄膜的成键特征进行分析。红外 光谱的背景是以未处理的铝基底偏振光分辨率为 $4 \mathrm{~cm}^{-1}$ 扫描 1024 次的条件下获得。利用微观摩擦磨 损试验机 (UMT-3, 美国 CETR 公司) 测定薄膜的 摩擦磨损性能。对偶件为轴承钢球, 直径 $4 \mathrm{~mm}$ 。实 验条件：室温 $20^{\circ} \mathrm{C}$ 左右，相对湿度保持在 $40 \% \sim 50 \%$, 往复行程 $6 \mathrm{~mm}$, 往复频率 $2 \mathrm{~Hz}$, 所施加载荷为 0.1 和 $0.5 \mathrm{~N}$ 。

\section{2 结果与讨论}

\section{1 薄膜的制备}

图 1 为氧化铝薄膜的 XRD 图谱。可以看出, 除 $2 \theta$ 值在 38.228、44.617、65.024、78.088 处的峰可 分别归结为铝的(111)、(200)、(220)和(311)晶面

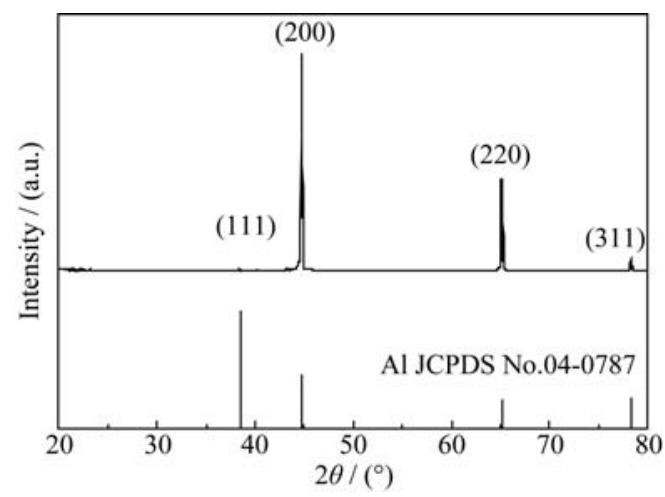

图 1 铝表面氧化铝薄膜的 XRD 图谱

Fig. 1 XRD pattern of alumina film on aluminum 
外，并无其他杂峰出现，说明铝表面的氧化铝薄膜 可能呈非晶态。

采用扫描电子显微镜对表面的微观形貌进行观 察。如图 2(a)所示, 未处理的铝表面除了部分加工 痕迹外表面十分光滑。图 2(b)给出铝表面氧化铝薄 膜的形貌照片，可以看出，表面存在颗粒凸点，但 是凸点分布不均，宏观的表面波纹度较高，同时表 面具有较多的细微皲裂纹和较宽的大裂纹。

由图 3 可以看出铝表面氧化铝薄膜的接触角测 量结果，薄膜呈超亲水性。

由于脂肪酸分子可吸附在材料表面并在摩擦过 程中与表面发生反应形成脂肪酸㿝膜 ${ }^{[27-29]}$, 本身具 有润滑和减摩作用，本研究使用脂肪酸改性氧化物 薄膜对氧化铝薄膜表面实现疏水化, 以制备出较理 想的摩擦学性能的薄膜。

表 1 为氧化铝薄膜经硬脂酸改性后的静态及动态 润湿性的检测结果, 可以看出, 硬脂酸改性的氧化铝 薄膜的静态接触角可达到 $144^{\circ}$, 但滚动角超过 $20^{\circ}$, 无 法达到超疏水状态。这说明, 水滴可能已完全润湿表面, 表面呈润湿 Wenzel 态。实验结果与文献[30]报道一致, 其主要原因在于溶胶一凝胶法生成的氧化铝薄膜较为 光滑, 缺乏实现超疏水特性所必要的表面微纳结构。

Tadanaga 等 ${ }^{[11-32]}$ 报道, 将溶胶一凝胶法制备的氧化 铝薄膜经沸水处理后, 采用全氟硅烷进行疏水化处理 得到的氧化铝薄膜表现出显著的超疏水特性。为此, 本 研究对铝表面的氧化铝薄膜进行了同样处理。图 4(a)
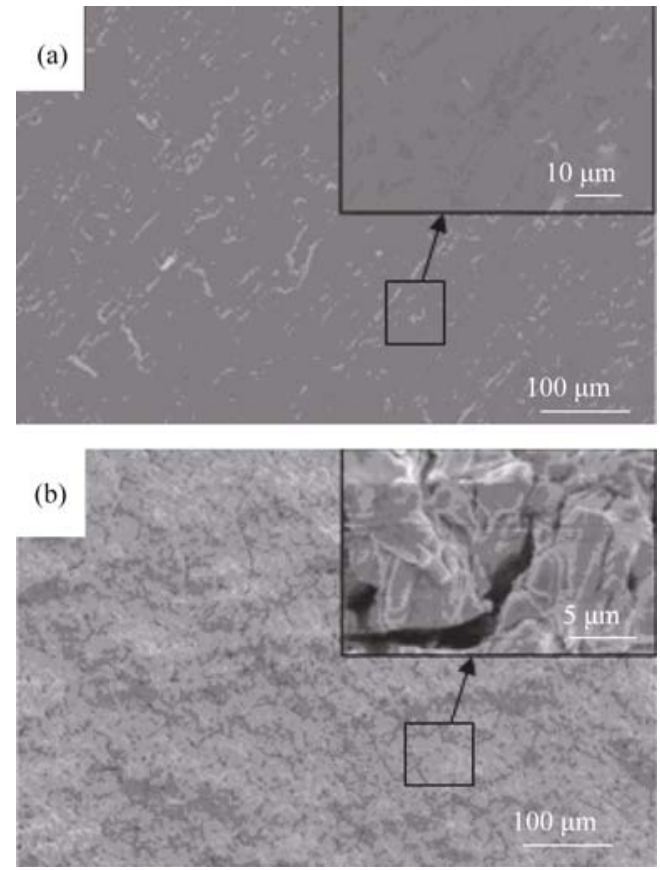

图 2 铝(a)及铝表面氧化铝薄膜(b)的 SEM 照片

Fig. 2 SEM images of aluminum (a) and alumina films on aluminum (b)

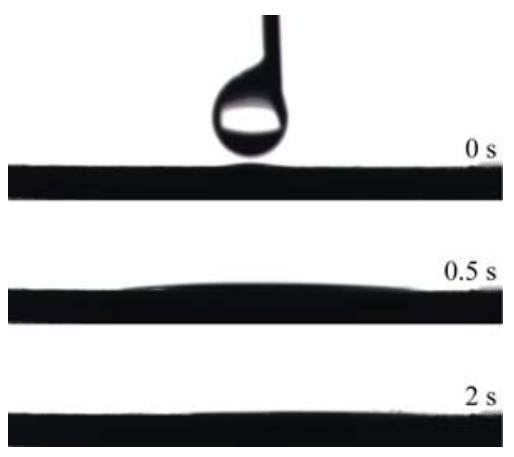

图 3 铝表面未处理氧化铝薄膜的动态润湿性

Fig. 3 Dynamic wettability of untreated alumina film on aluminum

表 1 硬脂酸改性不同氧化铝薄膜的润湿性

Table 1 Wetability of alumina films modified by stearic acid.

\begin{tabular}{lcc}
\hline \multicolumn{1}{c}{ Alumina film } & Contact angle $/\left({ }^{\circ}\right)$ Sliding angle $/\left({ }^{\circ}\right)$ \\
\hline Untreated & 144 & $>20.0$ \\
$\begin{array}{l}\text { Treated by boiling water } \\
\begin{array}{l}\text { Treated by hydrazine } \\
\text { hydrate }\end{array}\end{array}$ & 148 & 4.5 \\
\hline
\end{tabular}

为氧化铝薄膜经过沸水处理后的表面形貌照片，与未 处理的氧化铝薄膜(图 2(b))相比，宽大裂纹进一步增 大，同时观察到表面出现了氧化铝薄膜脱落形成的 “孔洞”，这可能是由于氧化铝与沸水相作用的结果。 使用硬脂酸对沸水处理过的氧化铝薄膜进行疏水化 处理, 如表 1 所示, 其静态接触角达到 $148^{\circ}$, 滚动角 下降到 $4.5^{\circ}$, 呈现出较为明显的疏水状态。
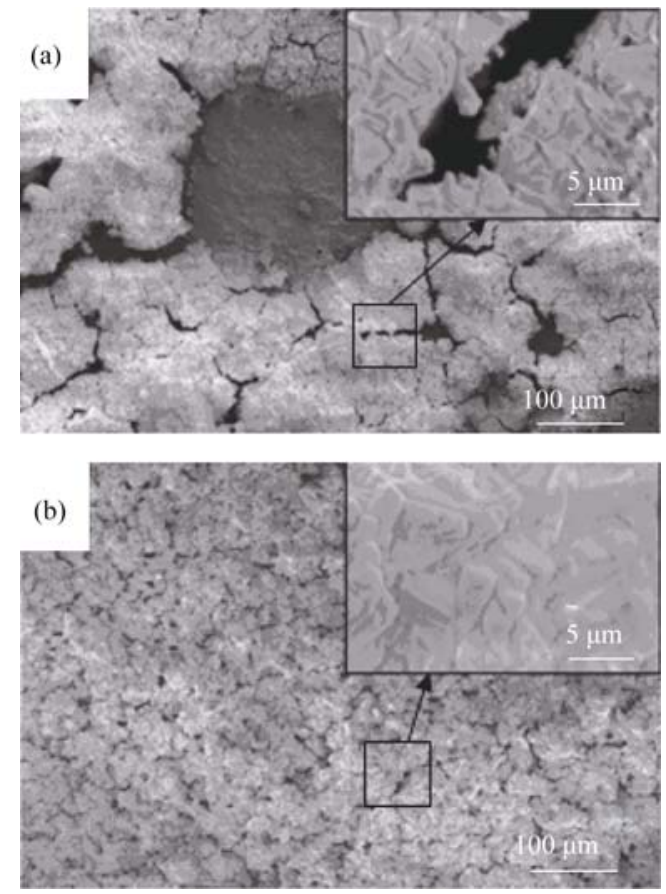

图 4 沸水(a)及水合肼溶液(b)处理氧化铝薄膜的 SEM 照片 Fig. 4 SEM images of alumina film treated by boiling water (a) and hydrazine hydrate (b) 
尽管沸水处理可以明显改善氧化铝薄膜的疏水 特性, 但也会对薄膜的整体结构产生影响, 这将影响 氧化铝薄膜的机械及摩擦学性能, 为此本研究探索采 用水合肼改性处理的新方法改善氧化铝薄膜的疏水 特性。图 4(b)给出经 $8 \%$ 的水合肼溶液处理后的氧化 铝薄膜的表面形貌, 与处理的氧化铝薄膜(图 2(b)) 相 比, 薄膜表面的裂纹完全消失。使用硬脂酸对水合肼 溶液处理的氧化铝薄膜进行疏水化处理, 如表 1 所示, 其接触角可达到 $150^{\circ}$, 滚动角为 $3^{\circ}$, 表现出良好的超 疏水状态。大的静态接触角和小的滚动角表明, 水滴 并没有渗入表面沟槽, 而仅停留在微纳结构表面。

采用傅里叶红外光谱仪对氧化铝薄膜表面脂肪 酸的成键特性进行的表征显示, 在氧化铝表面沉积 硬脂酸薄膜后, 样品在 $\mathrm{C}-\mathrm{H}$ 振动和 $\mathrm{C}-\mathrm{O}$ 振动区均存 在明显的吸收峰(图 5), 证明样品表面确实存在硬脂 酸薄膜, 这与前面接触角的观察数据相一致。根据以 往的研究, 如果脂肪酸在金属表面可形成有序薄膜, 烷链必为全反式结构, 即烷基链都以同一角度向表 面倾斜, 从而产生波数 $<2918 \mathrm{~cm}^{-1}$ 的特征吸收峰 ${ }^{[33]}$ 。 图 5 中 $2914 \mathrm{~cm}^{-1}$ 处的吸收峰正说明硬脂酸在铝表面 形成了有序薄膜。而对于低频区, 对比标准的硬脂酸 图谱不难看出, 在 $1700 \mathrm{~cm}^{-1}$ 处的吸收峰不再存在, $\mathrm{C}=\mathrm{O}$ 和 $\mathrm{C}-\mathrm{O}$ 键的振动峰位也发生了明显变化, 分别 偏移到 1584.8 和 $1471.5 \mathrm{~cm}^{-1}$ 处, 分别对应于硬脂酸 的自由羧基与样品表面羟基反应生成的酯键中碳氧 对称振动与非对称振动。说明在金属铝表面形成的 硬脂酸薄膜结构里的羧基已经发生变化, 薄膜中硬 脂酸的末端基团不再呈 $\mathrm{COOH}$ 结构, 而是以双配位 结构与表面发生作用 ${ }^{[34]}$ 。应该指出的是, 与水合肼 处理后的氧化铝表面的硬脂酸薄膜相比, 沸水处理 后的氧化铝薄膜表面经硬脂酸改性后, 尽管具有相 似的红外振动吸收, 但是在 $\mathrm{C}-\mathrm{H}$ 振动和 $\mathrm{C}-\mathrm{O}$ 振动区 的强度很低(图 5)。这表明经过水合肼处理后的氧化

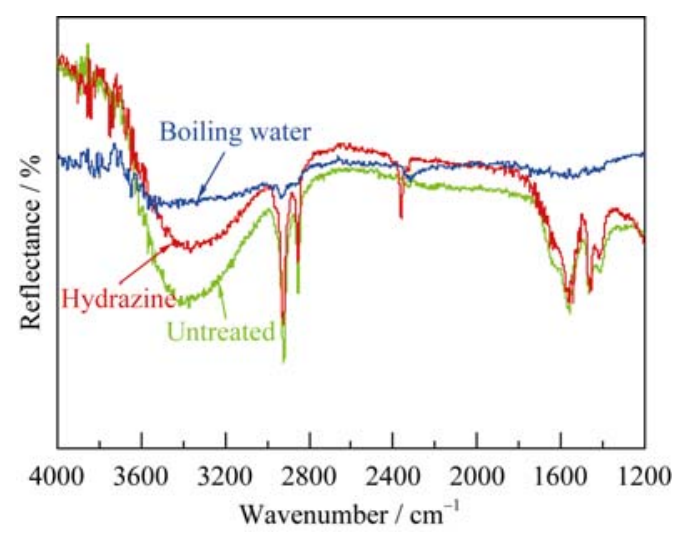

图 5 硬脂酸改性不同氧化铝薄膜的 IR 图谱

Fig. 5 IR spectra for steraic acid on various alumina films
铝薄膜更容易吸附硬脂酸, 同时“类荷叶”粗粘结构 可以容纳更多的硬脂酸分子, 促进了硬脂酸分子中 羧基与表面的反应, 从而有助于形成结构更为致密 的硬脂酸薄膜。

\section{2 薄膜的摩擦学性能}

采用球盘式试验机对所得样品的摩擦学性能进 行表征, 结果见图 6。在负载为 $0.1 \mathrm{~N}$ 下样品的摩擦 系数, 对于金属铝, 摩擦系数保持在 0.6 左右。而对 于铝基底上构筑的三种疏水氧化铝薄膜, 摩擦学性 能均得到了极大的改善, 其中以水合肼处理的氧化 铝薄膜表现出最好的摩擦学性能, 其摩擦系数稳定 在 0.14 左右, 减摩性能较强。而由于沸水处理破坏 了氧化铝薄膜的整体结构, 导致其摩擦学性能相对 较差。当负载升到 $0.5 \mathrm{~N}$ 时, 同样可测定出水合肼处 理的氧化铝薄膜表现出最好的减摩性能。

图 7 给出摩擦实验结束后磨痕的 SEM 照片, 相 比于未处理的氧化铝薄膜, 很难在水合肼处理的氧 化铝薄膜上观察到磨痕, 说明这层薄膜具有良好的 机械稳定性。而经沸水处理的氧化铝薄膜, 滑动过 程中, 氧化铝薄膜表面结构明显被破坏。

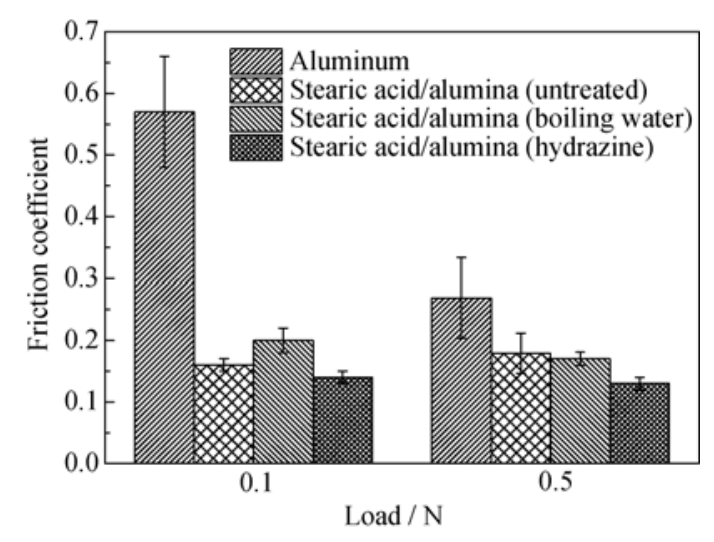

图 6 铝及硬脂酸改性氧化铝薄膜的摩擦系数

Fig. 6 Friction coefficients of aluminum and alumina film modified by stearic acid

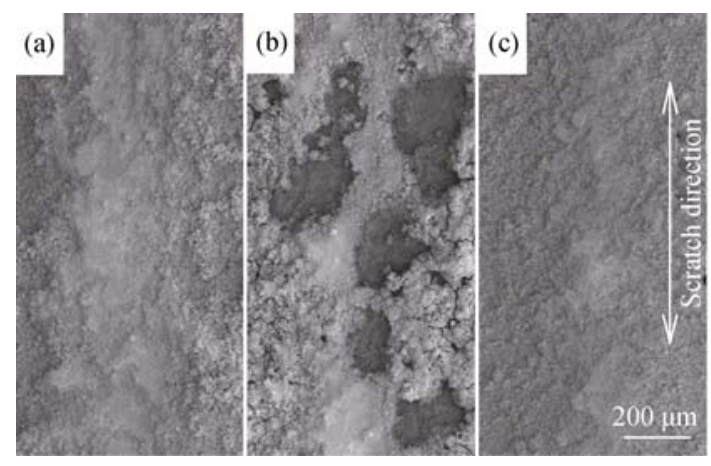

图 7 硬脂酸改性氧化铝薄膜的磨痕 SEM 照片

Fig. 7 SEM images for alumina films modified by stearic acid (a) Untreated alumina film; (b) Alumina film treated by boiling water; (c) Alumina film treated by hydrazine 
铝表面超疏水薄膜较强的减摩性能无疑与其 特殊的薄膜结构相关。已经证实, 在干摩擦条件下, 利用溶胶一凝胶技术在材料表面构筑的氧化铝薄膜 具有良好的机械强度, 可有效降低材料的磨损 ${ }^{[22]}$; 另一方面, 脂肪酸分子可吸附在材料表面并在摩 擦过程中与表面发生反应形成脂肪酸皇膜 ${ }^{[27-29]}$, 本身具有润滑和减摩作用。因此将上述两种技术结 合在铝表面构筑的超疏水薄膜，应该表现出更好 的摩擦学性能。同时, 超疏水薄膜较强的摩擦学性 能也与其独特的润湿性有关。以前 Bowden 等 ${ }^{[35]}$ 曾发现, 微纳条件下, 摩擦力与真实接触面积成正 比。而真实接触面积不仅依赖于正压力，而且更重 要是由界面能决定 ${ }^{[36]}$ 。由于材料静态接触角的余弦 值可作为衡量表面能大小的度量 ${ }^{[37]}$, 接触角越大 说明表面能越低，所以在金属铝表面构筑的超疏 水薄膜具有极低的表面能和相应更小的真实接触 面积, 因而表现出良好的减摩性能。

\section{3 脂肪酸结构对疏水薄膜的润湿及摩擦学 性能的影响}

选择与硬脂酸及与硬脂酸结构相类似的油酸 和月桂酸对未处理及水合肼处理的氧化铝薄膜进 行疏水改性后, 静态和动态润湿性结果显示于表 2 中, 摩擦学性能显示于图 8 中。可以看出, 在分子
链引入双键或减少碳链的长度均导致薄膜的疏水 性下降, 同时导致摩擦学性能变差。这主要是与脂 肪酸在氧化铝表面自组装薄膜的有序性以及堆积 密度有关 ${ }^{[38]}$ 。对于直链脂肪酸, 随碳链长度的增加, 形成的自组装薄膜更为致密，表面疏水性及相应 的摩擦学性能更好; 而在碳链引入双键则破坏了 自组装薄膜的有序性，导致润湿性及相应的摩擦 学性能变差。

\section{3 结论}

利用溶胶一凝胶技术在金属铝表面构筑的氧化 铝超疏水薄膜表现出较强的减摩耐磨性能, 特别 是经水合肼处理后并经硬脂酸改性后得到的超疏 水薄膜具有良好的减摩性能。金属铝表面超疏水薄 膜较强的减摩耐磨性能可归结为超疏水薄膜具有 极低的表面能，极大地减少了摩擦副之间的黏着， 同时也与硬脂酸在氧化铝表面形成更为致密的减 摩薄膜有关。

由此, 采取溶胶一凝胶技术在金属铝表面构筑的 超疏水表面表现出较强的减摩性能, 技术简单易行, 价格低廉, 易于工业化操作, 为改善在微纳条件下 铝及其合金的润滑提供了可能的途径。

表 2 不同样品的表面润湿性

Table 2 Wetability of various alumina films

\begin{tabular}{cccc}
\hline Alumina film & Hydrophobic agent & Contact angle $/\left(^{\circ}\right)$ & Sliding angle $/\left(^{\circ}\right)$ \\
\hline \multirow{2}{*}{ Untreated } & Stearic acid & 144 & $>20$ \\
& Lauric acid & 141 & $>90$ \\
& Oleic acid & 134 & $>90$ \\
Treated by hydrazine hydrate & Stearic acid & 150 & 3 \\
& Lauric acid & 146 & 5 \\
& Oleic acid & 143 & 9 \\
\hline
\end{tabular}
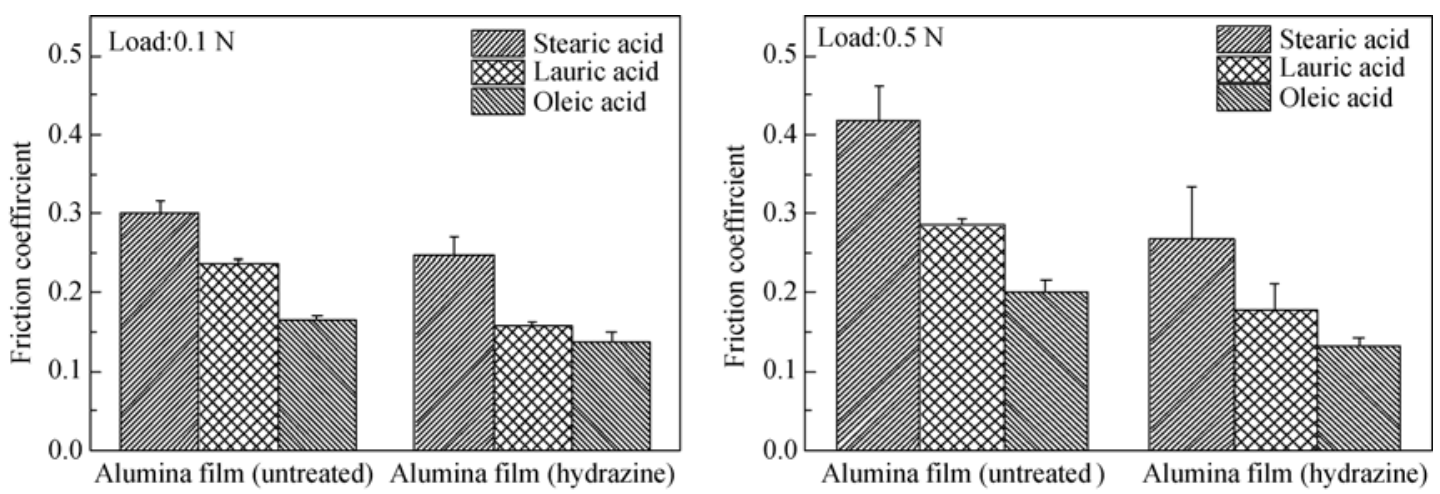

图 8 油酸、月桂酸或硬脂酸改性不同氧化铝薄膜的摩擦系数

Fig. 8 Friction coefficient for alumina films modified by oleic acid, lauric acid, or stearic acid 


\section{参考文献:}

[1] LIU W, XIA Y, XUE Q. Effect of P-N type extreme pressure and anti wear additive on the friction and wear behaviour of aluminum steel sliding pair. Tribology, 2000, 20(5): 331-335.

[2] HUANG W, YU Y, ZHANG X, et al. Effects of phosphorus additives on tribological performance of 7050 aluminum alloy. Chin. J. Nonferrous Metals, 2013, 23(3): 652-657.

[3] KONISHI T, PEREZ J M. Properties of polyol esters--lubrication of an aluminum silicon alloy. Tribol. Trans. 1997, 40(3): 500-506.

[4] SARMISTHA D, BISWAS S K. Boundary lubricated tribology of an aluminum-silicon alloy sliding against steel. Tribol. Lett., 2004, 17(3): 623-628.

[5] BHUSHAN B. Handbook of Nanotechnology. Springer: Heidelberg, 2010.

[6] MABOUDIAN R, CARRARO C. Surface chemistry and tribology of MEMS. Annu. Rev. Phys. Chem., 2005, 55(3): 35-54.

[7] WILliams J A, LE H R. Tribology and MEMS. J. Phys. D. Appl. Phys., 2006, 39(12): R201-R214.

[8] LIU L, SONG S Y, ZHANG P Y. Preparation and microtribological study on dually mixed self-assembled monolayers. Acta Phys. Chim. Sin., 2012, 28(2): 427-432.

[9] HOQUE E, DEROSE J A, HOFFMANN P, et al. Alkylperfluorosilane self-assembled monolayers on aluminum: a comparison with alkylphosphonate self-assembled monolayers. J. Phys. Chem. C, 2007, 111(10): 3956-3962.

[10] KHATRI O P, BAIN C D, BISWAS S K. Effects of chain length and heat treatment on the nanotribology of alkylsilane monolayers self-assembled on a rough aluminum surface. J. Phys. Chem. B, 2005, 109(49): 23405-23414.

[11] BARTHLOTT W, NEINHUIS C. Purity of the sacred lotus, or escape from contamination in biological surfaces. Planta, 1997, 202(1): 1-8.

[12] CHU Z, SEEGER S. Superamphiphobic surfaces. Chem. Soc. Rev., 2014, 43(8): 2784-2798.

[13] LIU X, LIANG Y, ZHOU F, et al. Extreme wettability and tunable adhesion: biomimicking beyond nature? Soft Matter, 2012, 8(7): 2070-2086.

[14] LIU K, JIANG L. Metallic surfaces with special wettability. Nanoscale, 2011, 3(3): 825-838.

[15] GUO Z, LIU W, SU B L. Superhydrophobic surfaces: from natural to biomimetic to functional. J. Colloid Interface Sci., 2011, 353(2): 335-355.

[16] ZHANG Y L, XIA H, KIM E, et al. Recent developments in superhydrophobic surfaces with unique structural and functional properties. Soft Matter, 2012, 8(44): 11217-11231.

[17] DI Z Y, HE J P, ZHOU J H, et al. Fabrication and anticorrosion property of superhydrophobic surfaces with hierarchical structure through an organic-inorganic self-assemble process. J. Inorg. Mater., 2010, 25(7): 765-769.

[18] RUAN M, LI W, WANG B, et al. Preparation and anti-icing behavior of superhydrophobic surfaces on aluminum alloy substrates. Langmuir, 2013, 29(27): 8482-8491.

[19] GHALMI Z, FARZANEH M. Durability of nanostructured coatings based on PTFE nanoparticles deposited on porous aluminum alloy. Appl. Surf. Sci., 2014, 314: 564-569.
[20] MENINI R, GHALMI Z, FARZANEH M. Highly resistant icephobic coatings on aluminum alloys. Cold Reg. Sci. Technol., 2011, 65(1): 65-69.

[21] ZHANG F, ZHAO L, CHEN H, et al. Corrosion resistance of superhydrophobic layered double hydroxide films on aluminium. Angew. Chem. Int. Ed. Eng., 2008, 47(13): 2466-2469.

[22] HUBERT T, SCHWARZ J, OERTEL B. Sol-Gel alumina coatings on stainless steel for wear protection. J. Sol-Gel Sci. Techn. 2006, 38(2): 179-184.

[23] ZHELUDKEVICH M L, MIRANDA SALVADO I, FERREIRAG M G S. Sol-Gel coatings for corrosion protection of metals. $J$. Mater. Chem., 2005, 15(4): 5099-5111.

[24] ZHENG S, LI J. Inorganic-organic Sol-Gel hybrid coatings for corrosion protection of metals. J. Sol-Gel Sci. Technol, 2010, 54(2): 174-187.

[25] LIU W, CHEN Y, LI B. Research progress on preparation and tribological investigation of Sol-Gel-derived ceramic-based ultrathin films. Tribology. 2003, 23(2): 162-167.

[26] FU Q, CAO C B, ZHU H S. Preparation of alumina films from a new Sol-Gel route. Thin Solid Films, 1999, 348(1/2): 99-102.

[27] BOWDEN F P, GREGORY J N, TABOR D. Lubrication of metal surfaces by fatty acids. Nature 1945, 156(3952): 97-101.

[28] SAHOO R R, BISWAS S K. Frictional response of fatty acids on steel. J. Colloid Interface Sci., 2009, 333(2): 707-718.

[29] RUTHS M, LUNDGREN S, DANERLÖV K, et al. Friction of fatty acids in nanometer-sized contacts of different adhesive strength. Langmuir, 2008, 24(4): 1509-1516.

[30] WU Y L, CHEN Z, ZENG X T. Nanoscale morphology for high hydrophobicity of a hard Sol-Gel thin film. Appl. Surf. Sci. 2008, 254(21): 6952-6958.

[31] YAMAGUCHI N, TADANAGA K, MATSUDA A, et al. Formation of anti-reflective alumina films on polymer substrates by the Sol-Gel process with hot water treatment. Surf. Coatings Technol. 2006, 201(6): 3653-3657.

[32] MA W, WU H, HIGAKI Y, et al. A "non-sticky" superhydrophobic surface prepared by self-assembly of fluoroalkyl phosphonic acid on a hierarchically micro/nanostructured alumina gel film. Chem. Commun., 2012, 48: 6824-6826.

[33] LAIBINIS P E, HICKMAN J J, WRIGHTON M S, et al. Orthogonal self-assembled monolayers: alkanethiols on gold and alkane carboxylic acids on alumina. Science, 1989, 245(4920): 845-847.

[34] TAO Y T. Structural comparison of self-assembled monolayers of n-alkanoic acids on the surfaces of silver, copper, and aluminum. $J$. Am. Chem. Soc. 1993, 115(10): 4350-4358.

[35] DOWSON D. History of tribology. London: Professional Engineering Publishing, 1998

[36] JOHNSON K L, KENDALL K, ROBERTS A D. Surface energy and the contact of elastic solids. Proc. R. Soc. London, Ser. A. 1971, 324(1558): 301-313.

[37] BEAKE B D, LEGGETT G I. Variation of frictional forces in air with the compositions of heterogeneous organic surfaces. Langmuir. 2000, 16(2): 735-739.

[38] CHENG H, HU Y. Influence of chain ordering on frictional properties of self-assembled monolayers (SAMs) in nano-lubrication. Adv. Colloid Interface Sci., 2012, 171-172: 53-65. 